
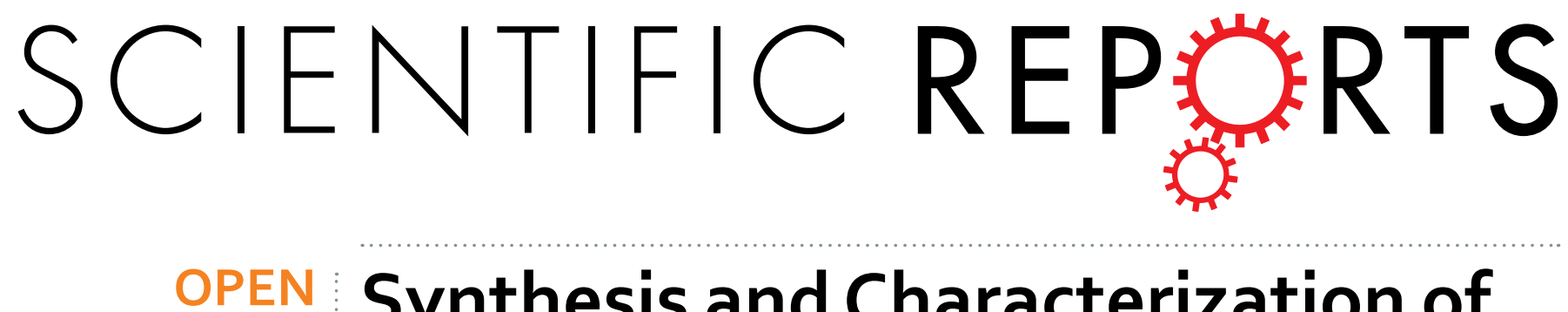

\title{
Synthesis and Characterization of Superhydrophobic, Self-cleaning NIR-reflective Silica Nanoparticles
}

Received: 16 August 2016

Accepted: 10 October 2016

Published: 08 November 2016

\section{Deepa Sriramulu ${ }^{1}$, Ella Louise Reed ${ }^{1}$, Meenakshi Annamalai ${ }^{2}$, Thirumalai Venky Venkatesan ${ }^{2,3,4,5,6}$ \& Suresh Valiyaveettil ${ }^{1}$}

Multifunctional coatings offer many advantages towards protecting various surfaces. Here we apply aggregation induced segregation of perylene diimide (PDI) to control the surface morphology and properties of silica nanoparticles. Differentially functionalized PDI was incorporated on the surface of silica nanoparticles through Si-O-Si bonds. The absorption and emission spectra of the resultant functionalised nanoparticles showed monomeric or excimeric peaks based on the amounts of perylene molecules present on the surface of silica nanoparticles. Contact angle measurements on thin films prepared from nanoparticles showed that unfunctionalised nanoparticles were superhydrophilic with a contact angle (CA) of $0^{\circ}$, whereas perylene functionalised silica particles were hydrophobic (CA $>130^{\circ}$ ) and nanoparticles functionalised with PDI and trimethoxy(octadecyl)silane (TMODS) in an equimolar ratio were superhydrophobic with static $\mathrm{CA}>150^{\circ}$ and sliding angle $(\mathrm{SA})<10^{\circ}$. In addition, the near infrared (NIR) reflectance properties of PDI incorporated silica nanoparticles can be used to protect various heat sensitive substrates. The concept developed in this paper offers a unique combination of super hydrophobicity, interesting optical properties and NIR reflectance in nanosilica, which could be used for interesting applications such as surface coatings with self-cleaning and NIR reflection properties.

Silica nanoparticles are used in medical, biological and chemical industries owing to their low toxicity, chemical inertness, optical transparency and biocompatibility ${ }^{1,2}$. Dye-doped silica nanoparticles are designed and tested for potential uses in biomedical and coating applications ${ }^{3,4}$ due to their highly desirable properties that include excellent photostability, tunable optical properties, controllable size, thermal and environmental stability and a large surface area to volume ratio ${ }^{5}$.

Perylene derivatives have been identified as fluorophores with high fluorescence quantum yield, good photostability ${ }^{6}$ and a high molar extinction coefficient $\left(10^{5} \mathrm{M}^{-1} \mathrm{~cm}^{-1} \text { for isolated perylene bisimide chromophores }\right)^{7}$ owing to a planar and highly conjugated structures. Furthermore, the high reflectance in the NIR region of the electromagnetic spectrum allows the incorporation of thermal insulating properties into materials ${ }^{8}$. Organic NIR absorbers have a wide range of applications such as invisible inks, NIR readable bar codes, laser-welding of plastics, laser induced thermal curing ${ }^{9}$, and energy saving applications ${ }^{10}$. The origin of the NIR reflectance is due to the high refractive index of the perylene derivatives incorporated on various coatings ${ }^{8,10}$. NIR fluorescent nanoparticles are useful as they provide negligible background fluorescence, low-inner-filtration interference in complex biological systems such as whole blood ${ }^{11}$ and used as substrates for the characterization of enzymatic processes $^{12}$.

The observed hydrophobicity of the lotus leaf surface has led to extensive research into the development of hydrophobic surfaces with hierarchal architectures ${ }^{13}$. For example, fabrics coated with dye incorporated silica nanoparticles were tested for the durability and wash fastness ${ }^{14}$, along with change in hydrophobicity with

${ }^{1}$ Department of Chemistry, 3 Science Drive 3, National University of Singapore (NUS), 117543, Singapore. ${ }^{2}$ NUSNNINanoCore, National University of Singapore, 117411, Singapore. ${ }^{3}$ Department of Physics, National University of Singapore, 117542, Singapore. ${ }^{4}$ Department of Electrical and Computer Engineering, National University of Singapore, 117576, Singapore. ${ }^{5}$ Department of Material Science and Engineering, National University of Singapore, 117575, Singapore. ${ }^{6}$ NUS Graduate School for Integrative Sciences \& Engineering, National University of Singapore, 117456, Singapore. Correspondence and requests for materials should be addressed to S.V. (email: chmsv@nus. edu.sg) 


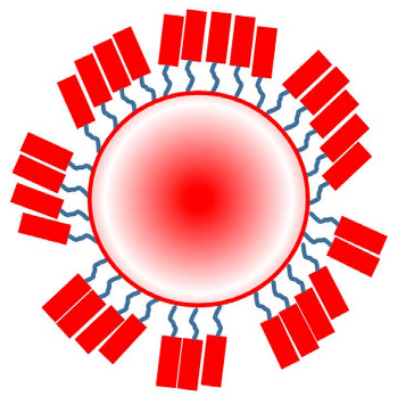

P-1, P-2, P-3

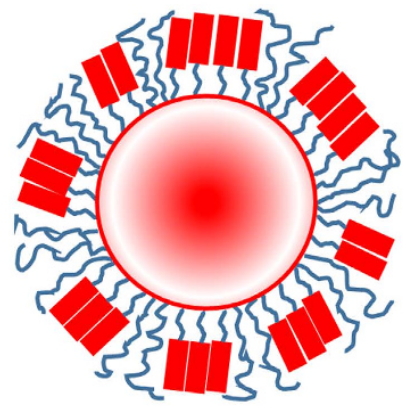

PT-1, PT-2

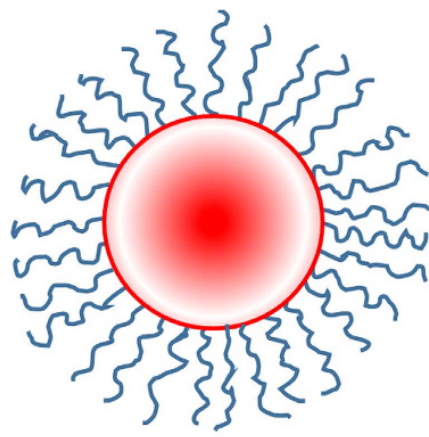

T1

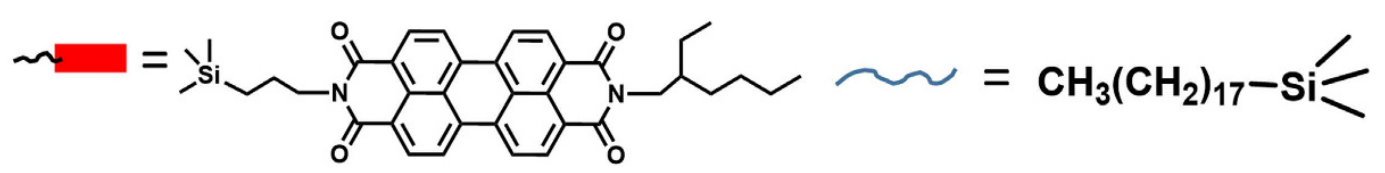

Figure 1. Cross sectional view of the conceptualized structures of the three silica nanoparticles prepared during this study. Full composition of each of these nanoparticle is given in the experimental section.

time ${ }^{15-17}$. Additionally, the degree of hydrophobicity to surface coating also reduced bacterial attachment leading to antimicrobial nanoparticles ${ }^{18}$.

Inorganic materials such as aluminum, zinc and titanium based metal oxides with NIR reflectance property and super hydrophobicity have been synthesized and reported in literature ${ }^{19,20}$. Organic fluorophores with high reflectance in the NIR region are rare and incorporation of these into a nanoparticle lattice offers many advantages such as low cost, biocompatiblity and easy accessibility. Consequently, the unique combination of hydrophobicity and NIR reflectance of such nanoparticles has led to the production of a diverse and multifunctional materials with interesting applications.

Herein, we explore the relationship between molecular organizations on the surface with the overall properties of the particle (Fig. 1). We report surface functionalization of silica nanoparticles with perylene derivatives and octadecyl groups to understand the significance of molecular arrangement on hydrophobic and optical properties of the particles. Three types of functionalised silica nanoparticles are used in this study, the first one involving particles with different concentrations of perylene molecules (P-1, P-2 and P-3), the second series with both perylene and octadecyl groups at different molar ratios (PT-1, PT-2) and the third one with only octadecyl groups (T1) on the surface. The questions addressed in this paper include, is it possible to control surface morphology using aggregation of perylene, which in turn influence the properties of the particles? Also, what would be the role of mixture of perylene and aliphatic long chain molecules towards the structure-property relationship of the nanoparticles? It is conceivable that flexible octadecylsilyl molecules fill the gaps between the perylene aggregates on the surface (PT-1, PT-2) and achieve complete coverage on the particle surface (Fig. 1).

\section{Results}

Synthetic Strategy and Characterization of Functionalised Silica Nanoparticles. Silica nanoparticles were synthesised using a reverse microemulsion method (see Supplementary Method S1) and fully characterised. Monodispersed silica nanoparticles were obtained with a narrow size distribution of around $40 \mathrm{~nm}$ (Fig. 2a).

PDI precursors were synthesised using a reported procedure ${ }^{21}$ (see Supplementary methods S2 and S3) and covalently attached to the surface of the silica nanoparticles. Different molar ratios of PDI and TMODS were functionalised onto the silica nanoparticle surface (Table 1). SEM images showed no difference in surface morphology before (pristine, Fig. 2a) and after functionalisation (P-3, Fig. 2b) on silica nanoparticles.

The FTIR spectra of the functionalised silica nanoparticles were recorded in the range of $500-4000 \mathrm{~cm}^{-1}$ in $\mathrm{KBr}$ matrix (Fig. 2c). Pristine silica nanoparticles were used as a control. The peaks observed at $3448 \mathrm{~cm}^{-1}$ and $1630 \mathrm{~cm}^{-1}$ corresponds to stretching vibrations of surface $\mathrm{O}-\mathrm{H}$ groups of the silica nanoparticles. The peaks $1096 \mathrm{~cm}^{-1}, 950 \mathrm{~cm}^{-1}$ and $795 \mathrm{~cm}^{-1}$ corresponds to $\mathrm{Si}-\mathrm{O}-\mathrm{Si}, \mathrm{Si}-\mathrm{OH}$ and $\mathrm{Si}-\mathrm{O}$ bonds, respectively. Compared to pristine silica nanoparticles, octadecyl groups incorporated particles PT-2 (1:1 molar ratio PDI to TMODS) and T1 showed additional peaks at $2929 \mathrm{~cm}^{-1}$ and $2855 \mathrm{~cm}^{-1}$ which correspond to $-\mathrm{CH}$ stretching vibration of alkyl groups. The perylene incorporated particles (P-1 to P-3 and PT-1, PT-2) showed peaks at $1691-1644 \mathrm{~cm}^{-1}$ for imide $\mathrm{C}=\mathrm{O}$ stretching and $1596 \mathrm{~cm}^{-1}$ for aromatic $\mathrm{C}=\mathrm{C}$ stretching vibrations. These results suggest that the perylene and octadecyl moieties were successfully functionalised onto the surface of the silica nanoparticles.

Thermogravimetric analyses of the functionalised silica nanoparticles were carried out under nitrogen atmosphere with a heating rate of $10^{\circ} \mathrm{C} / \mathrm{min}$ in the temperature range of $50-800^{\circ} \mathrm{C}$ (Fig. 3) and the results are summarised in Supplementary Table S1. All TGA traces exhibit a three stage degradation process. The first step of degradation was observed below $150^{\circ} \mathrm{C}$ corresponding to the loss of adsorbed water molecules. The second mass 

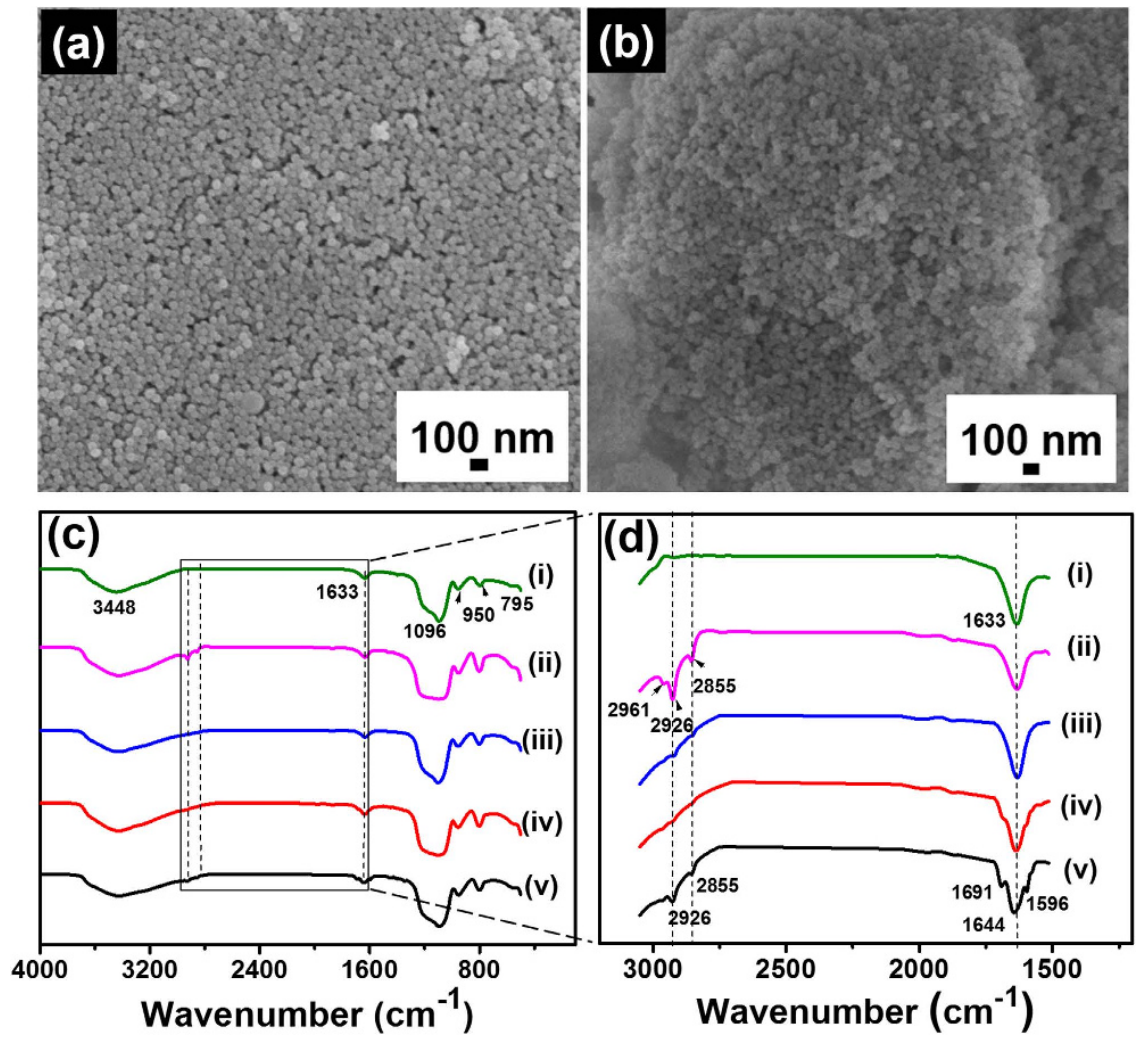

Figure 2. SEM image of monodispersed silica nanoparticles before (a) and after (b) functionalisation with perylene diimide molecules. FTIR spectra (c) of (i) $\mathrm{SiO}_{2}$ NPs, (ii) T1 (TMODS), (iii) P-1 (5 wt\% PDI), (iv) P3 (21 wt\% PDI), v) PT-2 (1:1 molar ratio of PDI to TMODS) functionalised silica nanoparticles. The marked region in (c) is expanded and given in (d) to show corresponding peaks clearly. IR spectra of P-2 and PT-1 NPs are given in the Supplementary Fig. S1a.

\begin{tabular}{|c|c|c|c|c|c|c|c|}
\hline Sample & $\begin{array}{r}\text { PDI } \\
(\mathrm{mg})\end{array}$ & $\begin{array}{c}\text { TMODS } \\
(\mu \mathrm{L})\end{array}$ & \% Ratio & $\begin{array}{c}\lambda_{\mathrm{abs}} \\
(\mathrm{nm})\end{array}$ & $\lambda_{\mathrm{emi}}(\mathbf{n m})$ & $\begin{array}{l}\text { Absorbance } \\
\text { ratio }\end{array}$ & $\begin{array}{c}\text { TGA (\%wt. } \\
\text { loss) }\end{array}$ \\
\hline P-1 & 5.0 & 0 & 5 & 523 & 534 & 1.04 & $6 \%$ \\
\hline P-2 & 10.0 & 0 & 10 & 490 & 530,628 & 0.99 & $5 \%$ \\
\hline P-3 & 21.0 & 0 & 21 & 494 & 650 & 0.78 & $8.2 \%$ \\
\hline PT-1 & 18.0 & 0.13 & $1: 0.01$ & 491 & 638 & 0.75 & $9 \%$ \\
\hline PT-2 & 18.0 & 12.8 & $1: 1$ & 490 & 630 & 0.89 & $11.3 \%$ \\
\hline $\mathrm{T} 1$ & - & 18.0 & 18 & - & - & - & $10.5 \%$ \\
\hline $\begin{array}{l}\text { PDI silane } \\
\text { precursor }\end{array}$ & - & - & - & 526 & 530 & $1.6^{*}$ & - \\
\hline
\end{tabular}

Table 1. Amount of reagents used for the synthesis of different batches of functionalized nanoparticles and the intensity ratios for the first and the second absorption peaks of perylene silica nanoparticles as compared to reference PDI silane precursor dissolved in THF. ${ }^{*}$ ) values from solution measurement, for all cases, $100 \mathrm{mg}$ of silica particles were used to start the reaction.

loss observed in the range of $150-400^{\circ} \mathrm{C}$, which can be assigned to the decomposition of organic groups and loosely bound $\mathrm{Si}-(\mathrm{OR})$ groups. The third mass loss observed in the range of $400-800^{\circ} \mathrm{C}$ was due to degradation of both the organosilicate framework and the PDI groups on the surface of the particles ${ }^{22}$. TMODS functionalised (T1) and PDI functionalised P-1, P-2 and P-3 nanoparticles showed a 4\%, 6\%, 6\% and $5 \%$ weight loss, respectively, below $150{ }^{\circ} \mathrm{C}$ as compared to the pristine silica nanoparticles ( $\left.8 \%\right)$. The observed weight loss for the functionalised silica nanoparticles can be explained by the fact that the surface functionalization of silica nanoparticles reduces the adsorption of water. When the temperature was further increased from $150-800^{\circ} \mathrm{C}$, T1 and P-3 nanoparticles showed a $10.5 \%$ and $8.2 \%$ weight loss, respectively, which can be explained as the loss of organic moieties grafted on the silica surface. Further, PT-1 and PT-2 showed weight loss of $9 \%$ and $\sim 11 \%$, respectively. These results are also supported by the elemental analysis (see Supplementary Table S1). 


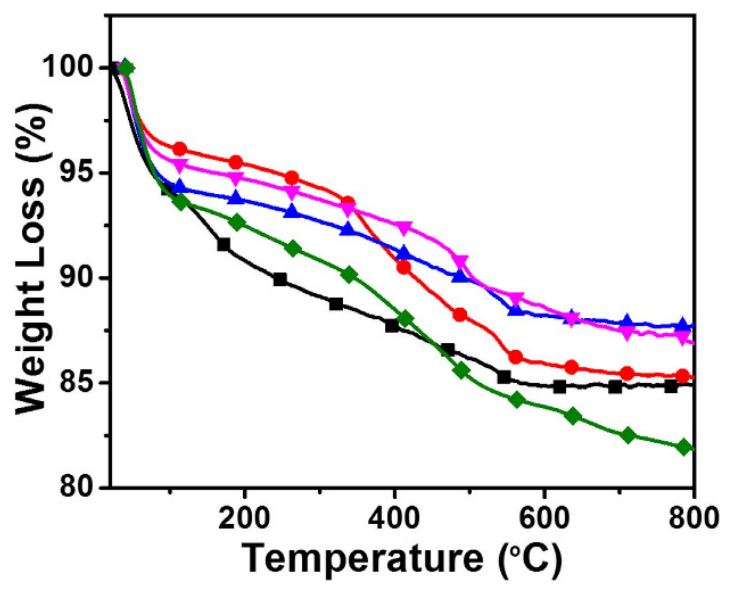

Figure 3. TGA traces of un-functionalised $(\boldsymbol{\square})$ and functionalised $(\boldsymbol{)}) \mathrm{T} 1,(\mathbf{\Delta})$ P-1, ( $\boldsymbol{\nabla})$ P-3, $(\diamond)$ PT-2 silica nanoparticles. Please see TGA traces of P-2 and PT-1 NPs in the Supplementary Fig. S1b.
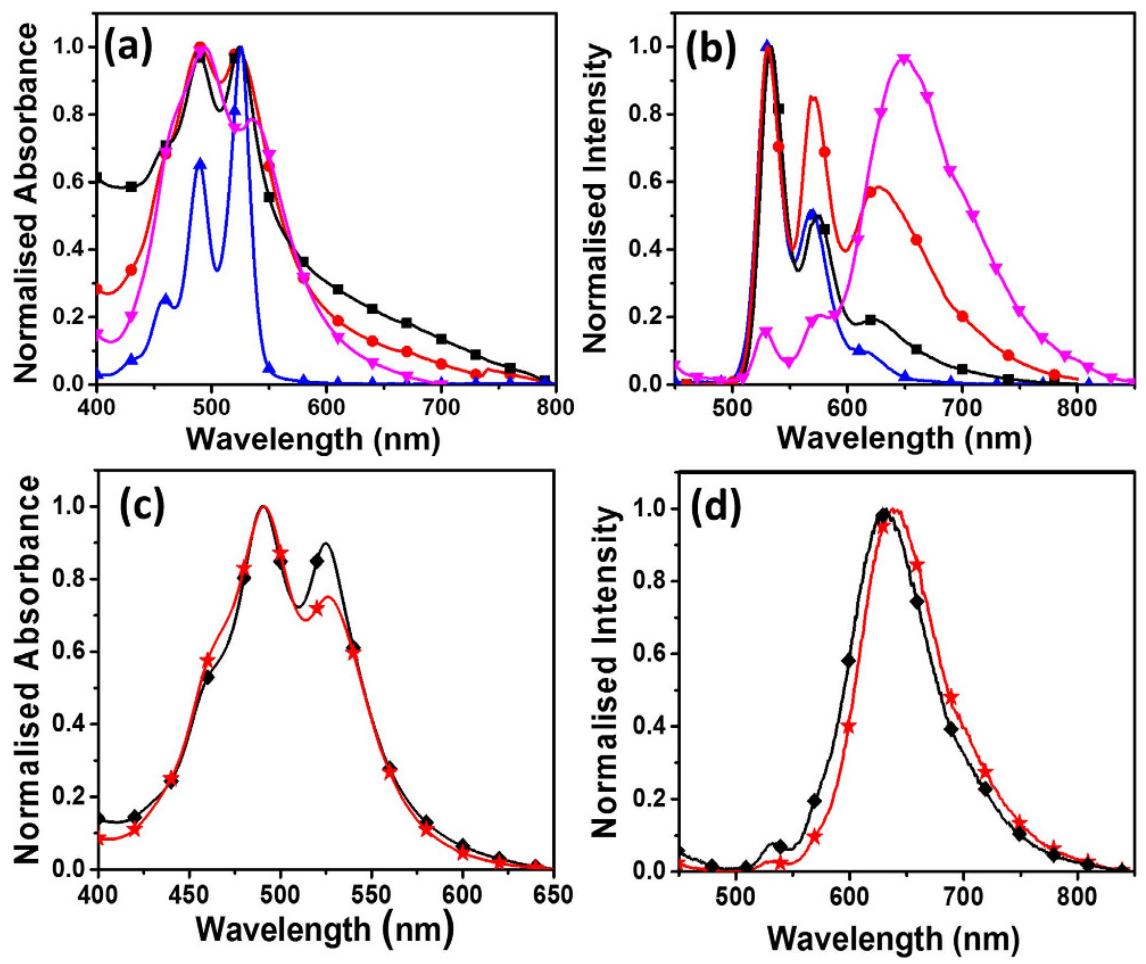

Figure 4. Normalised absorbance (a,c) and emission spectra (b,d) of (A) PDI silane precursor, ( $\mathbf{(}) \mathbf{P}-\mathbf{1}(5 \mathrm{wt} \%$ PDI), ( ) P-2 (10 wt\% PDI), ( $\nabla)$ P-3 (21 wt\% PDI), ( $\star$ ) PT-1 (1:0.01 molar ratio PDI to TMODS), ( $)$ PT-2 (1:1 molar ratio PDI to TMODS) functionalised silica nanoparticles dispersed in THF. Excitation wavelength used was $350 \mathrm{~nm}$.

Photophysical Properties of PDI Functionalised Silica Nanoparticles. The absorption and emission spectra of PDI and TMODS functionalised silica nanoparticles dispersed in THF are shown in Fig. 4.

The PDI silane precursors showed characteristic absorption peaks around 459, 489, and $526 \mathrm{~nm}$ corresponding to the $S_{0}-S_{1}$ transition with a well resolved vibronic structure from $0-0,0-1,0-2$ and $0-3$ transitions, respectively ${ }^{23}$. The absorption spectra of the PDI functionalized silica nanoparticles were broad with an inversion in peak intensities of $0-1$ and $0-0$ transition, compared to free PDI silane precursor in THF solution (Fig. 4a). The ratio of absorbance intensities of the $0-0$ and $0-1$ transitions are often used as an indication to determine the extent of aggregation ${ }^{24}$. The $\mathrm{A}_{0-0} / \mathrm{A}_{0-1}$ ratio from the absorption spectra of PDI silane precursor in THF was found to be $\sim 1.6$, which suggest the presence of free monomeric molecules in THF solution ${ }^{25}$. On functionalization of PDI onto the surface of the silica nanoparticles, the $\mathrm{A}_{0-0} / \mathrm{A}_{0-1}$ ratio was found to decrease with an increase in concentration of perylene on the surface and was 1.04 for P-1 (5 wt\% PDI), 0.99 for P-2 (10 wt\% PDI) and 0.79 (21 wt\% PDI) for P-3 nanoparticles. Strong vibronic coupling of $\mathrm{H}$-aggregates results in an increase in intensity 
of the (0-1) vibronic band relative to the (0-0) transition band. Based on the absorbance intensity ratio, the PDI molecules on the surface of the $\mathbf{P}-\mathbf{3}$ particles are more aggregated when compared to $\mathbf{P}-\mathbf{2}$ and $\mathbf{P}-\mathbf{1}$ particles. This is expected owing to the higher concentration of PDI molecules present on the surface. Introduction of PDI and TMODS in molar ratio of 1:0.01 (PT-1) and 1:1 (PT-2) led to changes in aggregation of the PDI molecules on the surface (Fig. 4c). The $\mathrm{A}_{0-0 /} \mathrm{A}_{0-1}$ ratio from the absorption spectra of the PT-2 was found to be 0.89 , slightly higher than that for PT-1 nanoparticles (0.75).

In order to understand the aggregation and arrangement of molecules on the surface, emission spectra of all particles were analyzed and compared with the solution spectra of PDI. The emission spectrum of PDI silane precursor in THF showed characteristic non-aggregated multiple maxima at 530, 568 and $618 \mathrm{~nm}$ (see Fig. 4b). Significant changes in the emission spectra of PDI molecules were observed after functionalization on the surface of silica nanoparticles (Fig. 4b). The nanoparticles with low concentration of PDI (P-1) showed an emission spectrum similar to the PDI silane precursor in solution. Upon increasing the concentration of PDI on the surface of the silica nanoparticles a new broad peak with a maximum around $650 \mathrm{~nm}$ was observed from P-3 nanoparticles, which is similar to aggregated PDI dyes in solution $(650 \mathrm{~nm})^{26}$. This suggests that the variations observed in the emission maxima of particles with different PDI concentrations is related to aggregation of molecules on the surfaces, which then allows fine tuning of the color of emission from yellowish green to bright pink (see Supplementary Fig. S2). The emission properties of the PDI on the surface of silica nanoparticles arise from the formation of distorted $\mathrm{H}$-aggregates with an increase in concentration ${ }^{27}$.

Interestingly, the PT-1 $\left(\lambda_{\text {emiss }}=638 \mathrm{~nm}, \Delta_{\text {P-3-PT-1 }}=12 \mathrm{~nm}\right)$ and PT-2 $\left(\lambda_{\text {emiss }}=630 \mathrm{~nm}, \Delta_{\text {P-3-PT-2 }}=20 \mathrm{~nm}\right.$, $)$ particles functionalized with both PDI and alkyl groups showed blue shift in emission peaks with respect to P-3 particles $\left(\lambda_{\text {emiss }}=650 \mathrm{~nm}\right)$ indicating higher distortion of $\mathrm{H}$-aggregates caused by the presence of long octadecylsilane groups (TMODS) on the surface as shown in Fig. $4 \mathrm{~d}$.

Characterization of Superhydrophobic properties. The wetting ability of surfaces coated with functionalised silica nanoparticles can be visualised by using highly polar substrates such as filter paper or silica-gel TLC plate (Fig. 5). The choice of the commercially available TLC plate was made to represent a highly hydrophilic rough surface. As expected, when the water droplet was placed on the unmodified commercially available silica TLC plate, complete absorption and spreading on the surface was observed indicating a superhydrophilic surface. In contrast, the $\mathbf{P}-3$ coated TLC plate surface was hydrophobic (CA 137.2 ${ }^{\circ}$, Fig. $5 \mathrm{a}$ ) and the PT-2 coated surface was superhydrophobic and repelled the water droplet completely (CA 156 $6^{\circ}$, Fig. 5b).

In order to further demonstrate the superhydrophobic effect of silica nanoparticles, a concentration gradient dependent hydrophobicity model was developed. One end of a long piece of filter paper of known length was dipped in THF solution of the dispersed PT-2 NPs to achieve a concentration gradient effect across the filter paper. The concentration gradient was formed through a combination of capillary force mediated wetting of the paper and movement of solution upward from the solution front, along with continued evaporation of the solvent THF from the surface of the paper. After drying at room temperature, the hydrophobicity was measured using water drop test method (Fig. 5c).

It was observed that lower part of the filter paper exhibited superhydrophobic character, where the water droplet completely rolled off from the surface of the coated filter paper when tilted (Fig. $5 \mathrm{~d}$ ). But other regions, where concentration of the nanoparticles are low, water droplet stayed on the surface even when the paper was tilted owing to the low concentration of the nanoparticles and strong interaction of water molecules with the paper surface. Finally, at the region where the surface is completely hydrophilic, water is absorbed upon contact. The changes in hydrophobicity and the wettability with water is directly proportional to the presence of PT-2 NPs on the surface of paper.

Superhydrophobicity depends on the surface energy and surface roughness of the sample. SEM images of the paper after coating of nanosilica (PT-2) showed uniform distribution of PT-2 nanoparticles on the surface of the paper (Fig. 5f), when compared to pristine filter paper (Fig. 5e). In general, superhydrophobicity is significantly enhanced when the hydrophobic surface has a hierarchal structure with a combination of both nanometre and micrometre-sized roughness ${ }^{28}$.

Contact angle measurements were performed using two types of substrates, namely, cleaned glass cover slip and silica TLC plate using goniometer measurements and summarised in Fig. 6a. Changes in contact angle were observed on glass surface when coated with functionalised silica nanoparticles. Bare clean glass surface provided a contact angle of only $\sim 55.7^{\circ}$, and coating with octadecyl silanes increased the contact angle to $\sim 105^{\circ}$. Coating with PT-2 NPs led to the formation of superhydrophobic surface with a water contact angle of $>150^{\circ}$. The increase in contact angle can be attributed to the formation of rough micro-nanomorphological pattern on these surfaces and reduction in surface energy of the particles.

Also, values of contact angle measured for the nanoparticle coated TLC plates were higher than the coated glass substrates. SEM micrographs of TLC plate showed random distribution of silica clumps of size around 8-22 $\mu \mathrm{m}$ with random pores (see Supplementary Fig. S3a) and coating of the functionalized silica nanoparticles led to a relatively less porous surface (see Supplementary Fig. S3b). As expected, commercially available TLC plate is superhydrophilic with complete absorption or spreading of water with a contact angle (CA) of $\sim 0^{\circ}$. Upon coating with functionalised silica nanoparticles, an increase in the contact angle value was observed similar to particles coated on paper. T1 particles showed a contact angle (CA) of $144^{\circ}$ and a sliding angle of $25^{\circ}$. Similarly, PDI functionalised silica NPs (P-3) coated TLC plate also showed a CA of $\sim 137^{\circ}$. Thus PT-2 coated TLC plates showed different behaviour as compared to T1 and P-3 coated TLC plates. This could be due to the arrangements of molecules on the surface of silica particles T1 and P-3, which could trap water droplets and thus reduces the contact angle (See Supplementary Fig. S4). PT-2 coated TLC plates exhibited non-sticky, water repellent properties with CA of $\sim 156^{\circ}$ and sliding contact angle (SA) less than $10^{\circ}$ indicating superhydrophobicity. 


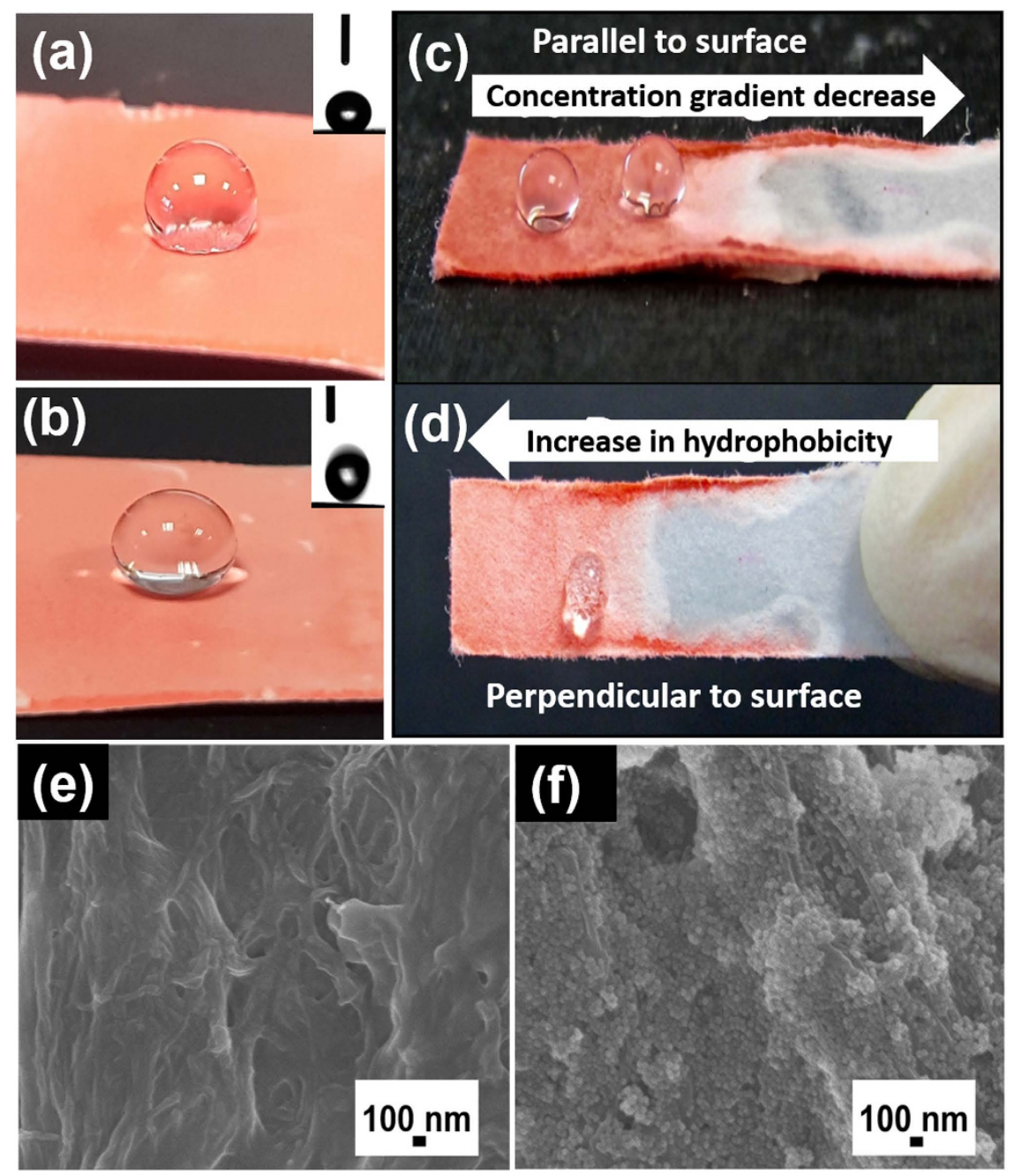

Figure 5. Image of water drop on the surface of (a) P-3, (b) PT-2 coated silica TLC plate. Inset shows corresponding image of water drop taken during contact angle measurements, where drop does not stick to the surface coated with PT-2 NPs. Effect of concentration gradient of PT-2 (1:1 molar ratio PDI to TMODS) silica nanoparticles coated on filter paper held parallel (c) and perpendicular (d) to the surface in the presence of water droplet. SEM images of paper before (e) and after (f) coating with PT-2 silica nanoparticles.

Video footage taken using a high speed camera was used to further demonstrate the water repellent properties of the nanoparticle coated surface. The kinetic energy of the droplet was transformed into vibrational energy on touching the PT-2 coated surface leading to the anisotropic bouncing of the water droplet without allowing it to rest in the Cassie state (Fig. 6b) ${ }^{29}$. As a control, the same experiment was performed with a T-1 (TMODS) coated surface (Fig. 6c). In contrast to the PT-2 system, when the water droplet fell on the T-1 coated surface it did not rebound from the surface.

The wettability of a material is often attributed to a low surface free energy and a high degree of roughness ${ }^{30}$. The 'Lotus effect' explains how hierarchal structures with micro- and nanoscale roughness can lead to superhydrophobicity and an extreme non-stick and water-repellent surface ${ }^{28}$. Higher magnification SEM images (see Supplementary Fig. S3b) of both P-3 and PT-2 coated TLC plates showed similar distribution of silica nanoparticles on the surface of TLC plate. Thus, hydrophobicity observed for all functionalised silica NPs (T1, P-3 and PT-2) coated TLC plates is due to micro-nanoroughness achieved by the assembly of functionalised silica nanoparticles on silica clusters. The distribution of nanoparticles may increase the amount of trapped air in between the particles, which then enhances the hydrophobicity, as described in the Cassie-Baxter model ${ }^{31}$. In addition, micro-nanoscale roughness was formed by the assembly of multiple layers of nanoparticles (Fig. 7a). Similar to the lotus leaf, the PT-2 coated surface possess a hierarchical structure from multiple layers of surface functionalised silica nanoparticles with hydrophobic groups (i.e. PDI and TMODS).

NIR reflectance of PDI functionalised nanoparticles. Approximately $52 \%$ of ultraviolet radiation that reaches the Earth is in the near infrared region $(700-2300 \mathrm{~nm})$ of the electromagnetic spectrum. Absorption of radiation in this region will eventually lead to heat gain in many objects ${ }^{32}$. As a counter measure, NIR reflective colorants are used as an effective means of reflecting such radiations. Since perylene derivatives have shown NIR reflectance ${ }^{8,10}$, it is conceivable that PDI functionalised silica nanoparticles will also exhibit such properties. 
(a)

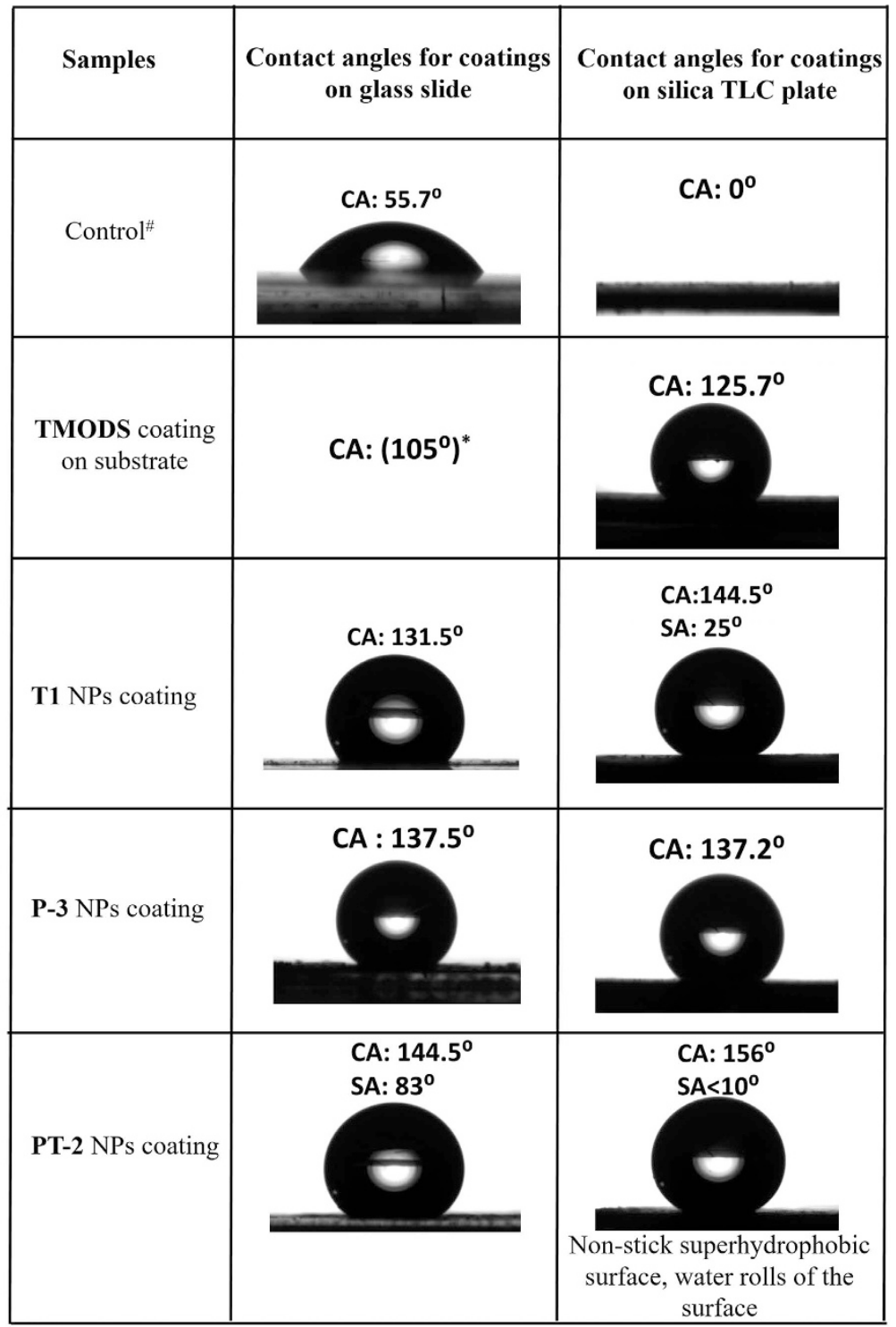

(b) $0.0 \mathrm{~ms}$

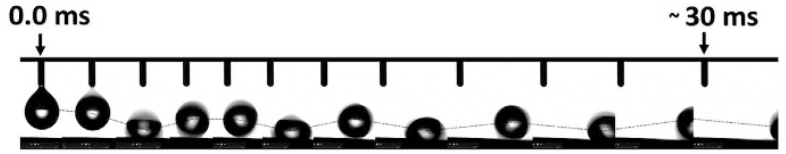

(c)

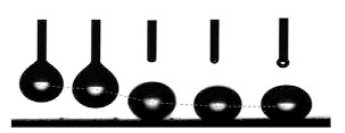

Figure 6. (a) Contact angle and sliding angles (CA and SA) of glass slide and silica TLC plate substrates coated with functionalised silica nanoparticles ( ${ }^{*}$ Control samples are bare glass cover slips and silica TLC plate, P-3, PT-2, functionalised silica nanoparticles, T1 refers to TMODS functionalised silica nanoparticles. ${ }^{\star}$ As per ref. 34). Selected stills showing the anisotropic bouncing of a water droplet on a PT-2 (b) and T1 (c) coated TLC plate in a $30 \mathrm{~ms}$ time frame.

The diffuse reflectance results obtained from PDI functionalised silica nanoparticles (P-3) coated on glass substrate using Teflon as a reference is shown in Fig. 7b. A narrow $1100 \mathrm{~nm}$ wavelength radiation was chosen for our experiment ${ }^{19}$. From the data obtained (Fig. 7c), PDI functionalised silica nanoparticles showed NIR reflectance up to $52 \%$ compared to pristine silica nanoparticles (28\%). Also, glass substrate coated with PDI showed maximum reflectance $(80 \%)$ in accordance with previous literature ${ }^{8,10}$. Such increase in NIR reflective properties after functionalisation of nanoparticle with PDI could be used to reduce the build-up of heat in materials from the absorption of NIR radiation. 

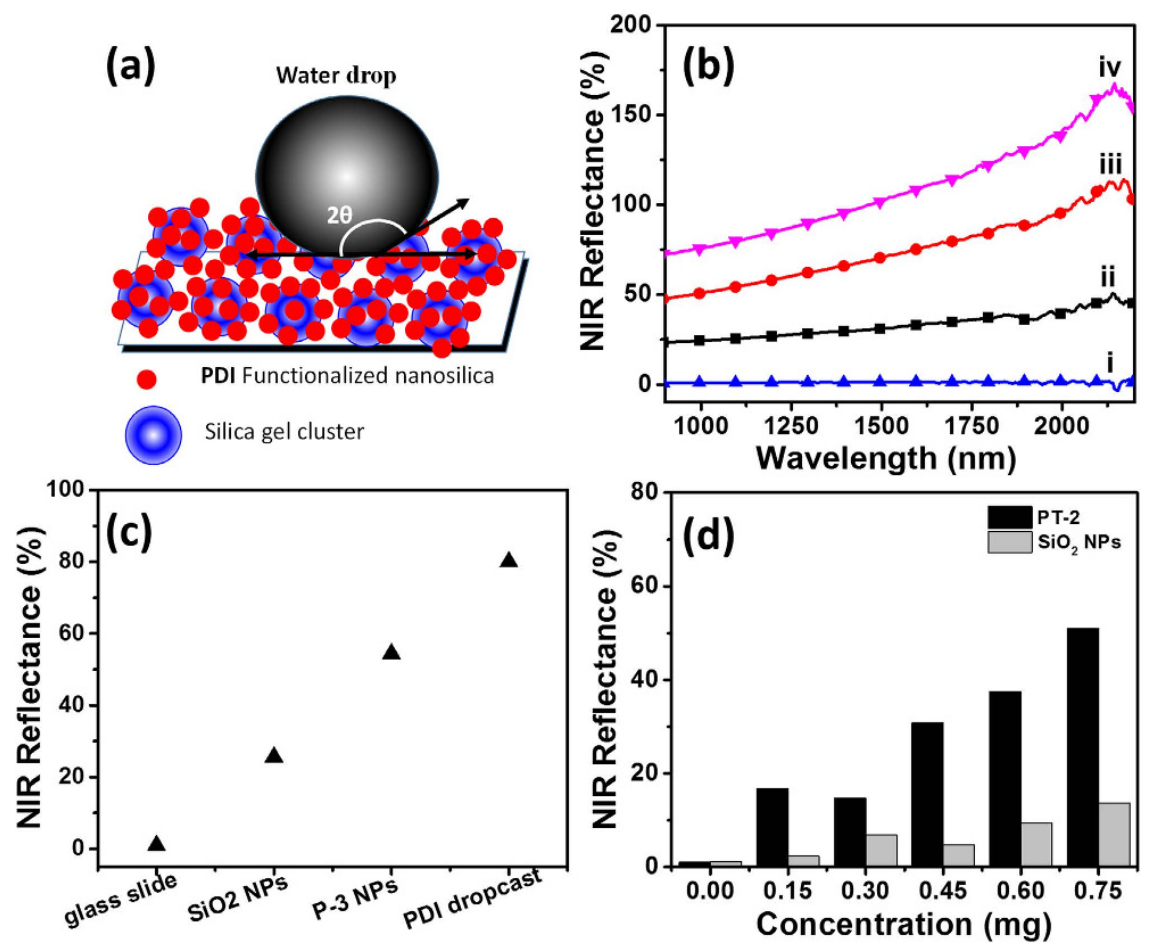

Figure 7. (a) Cartoonistic representation of water droplet on the surface of the PT-2 nanoparticle coated TLC surface. (Objects are not in scale). (b) \% NIR reflectance of (i) glass cover slip, (ii) unfunctionalised silica nanoparticles, (iii) P-3 (21 wt\% PDI), (iv) perylene diimide (PDI) drop cast on a glass cover slip in the range of 800-2200 nm. (c) The plot of (\%) NIR reflectance of different materials and (d) different concentrations of $\mathrm{SiO}_{2}$ and PT-2 (1:1 molar ratio PDI to TMODS) nanoparticles drop casted on glass cover slip.

The concentration dependent NIR reflectance studies were carried out on PT-2 silica nanoparticles and compared to pristine silica nanoparticles coated on a glass substrate as a control (Fig. 7d). The maximum reflectance (50\%) was obtained from PT-2 NPs compared to bare silica nanoparticles showing only $15 \%$ reflectance for the same amount $(0.75 \mathrm{mg})$ dispersed on glass cover slip. Further optimization of experimental variables such as the size of nanoparticles, concentration of PDI on the surface, coating processes, is currently in progress to optimize the NIR reflective properties. From the preliminary experiment, it is noted that PDI functionalised on silica nanoparticles do exhibit NIR reflective property.

\section{Discussion}

Six functionalised silica nanoparticles were synthesised, fully characterised and properties were investigated. The absorption and emission spectra of the samples showed correlation with the amount of perylene diimide (PDI) groups incorporated on the surface of the silica nanoparticles. Increasing the concentration of PDI, for example, led to a red shift in absorption maxima, changes in peak intensities and loss of vibrational fine structure demonstrating the aggregation induced formation of excimeric state. By using a mixture of PDI and TMODS on silica nanoparticle surface, the aggregation of PDI and organic molecule coverage on the particle surface were modulated. Among the six types of nanoparticles prepared, PT-2 showed the maximum coverage and fascinating properties. Pristine silica nanoparticles were hydrophilic $\left(\mathrm{CA}=0^{\circ}\right)$ and PDI functionalised nanoparticles showed hydrophobicity $\left(\mathrm{CA}=137.2^{\circ}\right)$. However, co-immobilization of the PDI and TMODS on the surface of particles led to superhydrophobic nanoparticles (PT-2) with a CA of $>150^{\circ}$ and a sliding contact angle $\left(\mathrm{SA}<10^{\circ}\right)$. The high hydrophobicity of the PT-2 is explained based on the effective coverage of the surface with organic molecules. Furthermore, the high NIR reflectance of PDI derivatives was used towards developing silica nanoparticle coatings with more than $80 \%$ NIR reflectance. In conclusion, here we report the successful synthesis of a fluorescent, superhydrophobic, self-cleaning NIR-reflective silica nanoparticles for coating on sensitive instruments.

\section{Methods}

Materials. Tetraethylorthosilicate (TEOS), (3-aminopropyl)triethoxysilane (APTES), perylene-3,4,9,10-tetracarboxydianhydride and trimethoxy(octadecyl)silane (TMODS) were obtained from Sigma Aldrich. Aqueous ammonia solution (25\%) was obtained from Merck. All chemicals and solvents used were AR grade and used without further purification.

Characterization Methods. Nuclear magnetic resonance $\left({ }^{1} \mathrm{H}\right.$ NMR) spectra were recorded on Bruker Avance AV300 (300 MHz) NMR instrument using $\mathrm{CDCl}_{3}$ as the solvent. Absorption and emission spectra were measured on a UV-1601PC Shimadzu spectrophotometer and RF-5301PC Shimadzu spectrofluorophotometer. 
Bruker ALPHA FT-IR spectrophotometer was used for records Fourier transform infrared (FT-IR) spectroscopy analysis. Thermogravimetric analyses (TGA) were conducted using a SDT 2960 TA instrument. All samples were heated under nitrogen atmosphere from $25^{\circ} \mathrm{C}$ to $800^{\circ} \mathrm{C}$ using a heating rate of $10^{\circ} \mathrm{C} / \mathrm{min}$. FT-IR spectra were recorded in the range of 3800-400 $\mathrm{cm}^{-1}$ using a Varian Excalibur 3100. Samples were mixed with $\mathrm{KBr}$ powder and grounded in an agate mortar before being pressed into a disk for recording the spectrum.

Contact Angle Measurement. The macroscale contact angle measurements were carried out using the video-based fully automated data physics optical contact angle microlitre dosing system (OCA 40 Micro, Germany). Deionised water drops $(1 \mu \mathrm{l} / \mathrm{drop})$ with known surface tension were dispensed using Teflon coated motor driven syringe. The contact angles were measured at be measured at $22^{\circ} \mathrm{C}$ and $45 \% \mathrm{RH}$ conditions and a video was recorded ( 72 frames/second) for every dispensed solvent droplet. Any dynamic changes to the droplet on the surface could be precisely observed through this method ${ }^{33}$.

Functionalised silica nanoparticles $(0.005 \mathrm{~g})$ were dispersed in THF $(2 \mathrm{ml})$ and sonicated for 10 minutes. TLC plates were dip coated in the dispersion of silica nanoparticles in THF and left to dry at room temperature. This procedure was repeated several times until a uniform coating was achieved.

For coating on glass cover slips, aqueous $\mathrm{NaOH}$ solution (10\%) was used to clean the surface and dried prior to drop casting the solution of silica nanoparticles in THF.

Finally, a gradient coating of functionalized silica nanoparticles on a strip of filter paper $(0.5 \times 1.0 \mathrm{~cm})$ was achieved by touching one end of the paper to the nanoparticle solution and letting the capillary action draw the solution to the paper. During the rise of solution through the paper, the solvent THF tends to evaporate and deposit the particles on the paper. This process allowed us to create a gradient with more nanoparticles at the end of the paper closer to the solution and less particles at the other end. Since the nanoparticles are red in color, the capillary gradient coating is visible with the naked eye. Three sections with high, medium and low concentrations of nanoparticles on coated filter paper were then tested to demonstrate how the degree of hydrophobicity was dependent on concentration of the particles adsorbed.

NIR Reflectance Measurement. The NIR diffuse reflectance of the silica nanoparticles coated glass substrate was measured using UV-Vis-NIR spectrophotometer (Shimadzu, UV-3600) with an integrating sphere attachment, ISR-3100. The NIR reflectance measurements of the samples were performed within the wavelength range of $700-2200 \mathrm{~nm}$.

General Procedure for Functionalization of Silica Nanoparticles. Silica nanoparticles (100 mg) dispersed in dry toluene $(10 \mathrm{ml})$ under nitrogen atmosphere were treated with PDI silane precursors and/or TMODS at different ratios to functionalise the surface (see Table 1). The mixture was refluxed under constant stirring at $90^{\circ} \mathrm{C}$ for 24 hours, centrifuged to obtain the solid and washed with THF to remove unreacted silane precursors before drying.

A summary of the amounts of reagents used for the synthesis of functionalised nanoparticles is given in Table 1, where P-1, P-2 and P-3 refers to 5, 10 and 21 wt\% of PDI, respectively, in solution, and PT-1 and PT-2 refers to a 1:0.01 and a 1:1 molar ratio of PDI to TMODS reagents, respectively, and T1 refers to silica nanoparticles functionalized with TMODS only.

\section{References}

1. Knopp, D., Tang, D. \& Niessner, R. Bioanalytical applications of biomolecule-functionalized nanometer-sized doped silica particles. Anal. Chim. Acta. 647, 14-30 (2009)

2. Montalti, M., Battistelli, G., Cantelli, A. \& Genovese, D. Photo-tunable multicolour fluorescence imaging based on self-assembled fluorogenic nanoparticles. Chem. Soc. Rev. 50, 5326-5329 (2014).

3. Ruedas-Rama, M. J., Walters, J. D., Orte, A. \& Hall, E. A. H. Fluorescent nanoparticles for intracellular sensing: A review. Anal. Chim. Acta. 751, 1-23 (2012).

4. Hong Nhung, T. et al. Dye-doped silica-based nanoparticles for bioapplications. Adv. Nat. Sci.: Nanosci. Nanotechnol. 4, 043001 (2013).

5. Sreenivasan, V. K. A., Zvyagin, A. V. \& Goldys, E. M. Luminescent nanoparticles and their applications in the life sciences. J. Phys. Condens. Matter. 25, 194101 (2013).

6. Ma, Y. et al. A water-soluble fluorescent $\mathrm{pH}$ probe based on perylene dyes and its application to cell imaging. Luminescence. 31, 102-107 (2016)

7. Gallaher, J. K., Aitken, E. J., Keyzers, R. A. \& Hodgkiss, J. M. Controlled aggregation of peptide-substituted perylene-bisimides. Chem. Commun. 48, 7961-7963 (2012).

8. Kaur, B., Bhattacharya, S. N. \& Henry, D. J. Interpreting the near-infrared reflectance of a series of perylene pigments. Dyes Pigm. 99, 502-511 (2013).

9. Qian, G. \& Wang, Z. Y. Near-infrared organic compounds and emerging applications. Chem. Asian J. 5, 1006-1029 (2010).

10. Kaur, B., Quazi, N., Ivanov, I. \& Bhattacharya, S. N. Near-infrared reflective properties of perylene derivatives. Dyes Pigm. 92, 1108-1113(2012).

11. Deng, T., Li, J., Jiang, J., Shen, G. \& Yu, R. Preparation of near-IR fluorescent nanoparticles for fluorescence-anisotropy-based immunoagglutination assay in whole blood. Adv Funct Mater. 16, 2147-2155 (2006).

12. Freschauf, L. R., McLane, J., Sharma, H. \& Khine, M. Shrink-induced superhydrophobic and antibacterial surfaces in consumer plastics. Plos One 7, e40987 (2012).

13. Parhizkar, M., Zhao, Y., Wang, X. \& Lin, T. Photostability and durability properties of photochromic organosilica coating on fabric. J. Eng. Fiber. Fabr. 9, 65-73 (2014).

14. Liu, B. et al. Synthesis and characterization of photoreactive silica nanoparticles for super-hydrophobic cotton fabrics application. Text. Res. J. 85, 795-803 (2015).

15. Hirashima, H., Imai, H. \& Fukui, Y. Structure of Hybrid Silica Gels Incorporated with Hydrophobic Dye Molecules. J. Sol-Gel Sci. Technol. 26, 383-388 (2003).

16. Lin, J., Chen, H., Fei, T. \& Zhang, J. Highly transparent superhydrophobic organic-inorganic nanocoating from the aggregation of silica nanoparticles. Colloids Surf., A. 421, 51-62 (2013). 
17. Lee, J. H., Park, E. J., Kim, D. H., Jeong, M. G. \& Kim, Y. D. Superhydrophobic surfaces with photocatalytic activity under UV and visible light irradiation. Catal. Today. 260, 32-38 (2016).

18. Weir, E., Lawlor, A., Whelan, A. \& Regan, F. The use of nanoparticles in anti-microbial materials and their characterization. Analyst. 133, 835-845 (2008).

19. Soumya, S., Mohamed, A. P., Mohan, K. \& Ananthakumar, S. Enhanced near-infrared reflectance and functional characteristics of Al-doped ZnO nano-pigments embedded PMMA coatings. Sol. Energ. Mat. Sol. Cells. 143, 335-346 (2015).

20. Xu, Q. F., Liu, Y., Lin, F. J., Mondal, B. \& Lyons, A. M. Superhydrophobic TiO2-Polymer Nanocomposite Surface with UV-Induced Reversible Wettability and Self-Cleaning Properties. ACS Appl. Mater. Interfaces 5, 8915-8924 (2013).

21. Huang, H., Che, Y. \& Zang, L. Direct synthesis of highly pure perylene tetracarboxylic monoimide. Tetrahedron Lett. 51, 6651-6653 (2010).

22. Wahab, M. A., Hussain, H. \& He, C. Photoactive Perylenediimide-Bridged Silsesquioxane Functionalized Periodic Mesoporous Organosilica Thin Films (PMO-SBA15): Synthesis, Self-Assembly, and Photoluminescent and Enhanced Mechanical Properties. Langmuir 25, 4743-4750 (2009).

23. Ribeiro, T., Baleizão, C. \& Farinha, J. P. S. Synthesis and Characterization of Perylenediimide Labeled Core-Shell Hybrid SilicaPolymer Nanoparticles. J. Phys. Chem. C. 113, 18082-18090 (2009).

24. Li, X., Sinks, L. E., Rybtchinski, B. \& Wasielewski, M. R. Ultrafast aggregate-to-aggregate energy transfer within self-assembled lightharvesting columns of zinc phthalocyanine tetrakis(perylenediimide). J. Am. Chem. Soc. 126, 10810-10811 (2004).

25. Gómez, R. et al. Energy and electron transfer in a poly(fluorene-alt-phenylene) bearing perylenediimides as pendant electron acceptor groups. Macromolecules 40, 2760-2772 (2007).

26. Neuteboom, E. E., Meskers, S. C. J., Meijer, E. W. \& Janssen, R. A. J. Photoluminescence of Self-organized Perylene Bisimide Polymers. Macromol. Chem. Phys. 205, 217-222 (2004).

27. Chen, Z., Baumeister, U., Tschierske, C. \& Würthner, F. Effect of core twisting on self-assembly and optical properties of perylene bisimide dyes in solution and columnar liquid crystalline phases. Chem. Eur. J. 13, 450-465 (2007).

28. Barthlott, W. \& Neinhuis, C. Purity of the sacred lotus, or escape from contamination in biological surfaces. Planta. 202, 1-8 (1997).

29. Huang, J. Y. et al. Robust superhydrophobic TiO2@fabrics for UV shielding, self-cleaning and oil-water separation. J. Mater. Chem. A. 3, 2825-2832 (2015)

30. Terpiłowski, K., Rymuszka, D., Goncharuk, O. V., Sulym, I. Y. \& Gun'ko, V. M. Wettability of modified silica layers deposited on glass support activated by plasma. Appl. Surf. Sci. 353, 843-850 (2015).

31. Chu, Z. \& Seeger, S. Superamphiphobic surfaces. Chem. Soc. Rev. 43, 2784-2798 (2014).

32. Levinson, R., Berdahl, P. \& Akbari, H. Solar spectral optical properties of pigments - Part I: Model for deriving scattering and absorption coefficients from transmittance and reflectance measurements. Sol. Energ. Mat. Sol. Cells. 89, 319-349 (2005).

33. Annamalai, M. et al. Surface energy and wettability of van der Waals structures. Nanoscale 8, 5764-5770 (2016).

34. Wu, Y. et al. Patterned hydrophobic-hydrophilic templates made from microwave-plasma enhanced chemical vapor deposited thin films. Thin Solid Films. 515, 4203-4208 (2007).

\section{Acknowledgements}

The authors acknowledge funding support from National University of Singapore and technical support from Department of Chemistry, National University of Singapore. Deepa Sriramulu thanks Ministry of Education and National University of Singapore for a research scholarship towards her PhD program.

\section{Author Contributions}

D.S. and E.R. conducted experiments, wrote manuscript and analyzed results; S.V. designed, analyzed results and revised manuscript; M.A. and T.V. conducted contact angle measurement. All authors reviewed manuscript.

\section{Additional Information}

Supplementary information accompanies this paper at http://www.nature.com/srep

Competing financial interests: The authors declare no competing financial interests.

How to cite this article: Sriramulu, D. et al. Synthesis and Characterization of Superhydrophobic, Self-cleaning NIR-reflective Silica Nanoparticles. Sci. Rep. 6, 35993; doi: 10.1038/srep35993 (2016).

Publisher's note: Springer Nature remains neutral with regard to jurisdictional claims in published maps and institutional affiliations.

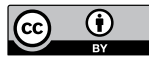

This work is licensed under a Creative Commons Attribution 4.0 International License. The images or other third party material in this article are included in the article's Creative Commons license, unless indicated otherwise in the credit line; if the material is not included under the Creative Commons license, users will need to obtain permission from the license holder to reproduce the material. To view a copy of this license, visit http://creativecommons.org/licenses/by/4.0/

(c) The Author(s) 2016 